\title{
A NEW GRAPHICAL METHOD OF CALCULATING LEADS FOR WIRING.
}

BY CARL HERING.

The subject of the calculation of leads for wiring has been written about in periodicals and books so often that it may almost be necessary to preface this paper with an excuse for again bringing up at this meeting such a simple and threadbare subject. The calculation of a single pair of leads is such a simple matter that very little need or can be said about it; the formula, if not at hand, can be deduced about as quickly as it could be looked up. But simple as this calculation is, if it has to be made not only tens, but hundreds of times, a method which shortens this work may perhaps be of intere.t to those whose misfortune it is to have much of this uninteresting work to do. The following suggestions of a graphical method is an attempt to reduce this work to what might be called " machine methods," by substituting for the usual figuring, a simple graphical device which requires no figuring at all and which is at the same time universal in its application, as it is adapted equally well for any of the many different lamps in the market to-day.

Makers and users of one particular lamp can readily calculate a wiring table for that lamp, for each of a few of the usual losses in the leads, and such tables are often quite convenient for those particular conditions; but it is evident that they are limited to those fixed conditions. Their scope can be increased by making simple reductions, but they may then become dangerous tools in the hands of many mechanics who may readily fall into such errors as multiplying instead of dividing by two. Furthermore, such tables are not generally applicable to branched leads, which are 
so common in wiring, and mechanics (they cannot be called electricians) have frequently made the error of determining from the tables each successive section of a branched wire for the total loss. It is no wonder, then, that they can underbid their competitors. To make a set of tables large enough to cover such fractional losses means to multiply their number consideral)ly. If in addition to this. such a set of tables is to include all the many different lamps of different makes and candle powers, it is readily seen that the number of tables which become necessary would be greatly burdened by the searches for the special table required for each caleulation. As long as there are lamps of such different amperage and voltage, it would hardly be practicable to make a universal set of tables, especially when the currents which the lamps take are greater than what is claimed for them.

Graphical methods, therefore, suggest themselves, for in these the size of the diagrams does not increase nearly as rapidly for an increased scope as the number of tables would. Graphical methods have been devised to replace the tables, but they have not been satisfactory for various reasons. As early as 1884 a diagra:n was devised by Emil Waldstrom, which was universil in its scope and exceedingly ingenious. It was based on logarithmic functions and gave not only the cross-section, but also the diameter of the wire, for any lamps, distances or losses. But the dense network of lines, and the method of using it, were so complieated that only technically trained engineers would be likely to apply it in practice. In the same year the writer devised and used a diagram totally different in principle, which had the advantage over the other in being so simple that it could readily be understuod; in fact, it could not be misunderstood, as there was only one set of diagonal lines. It consisted merely of a right angle and a series of diagonal. lines drawn through the centre, the background being cross-hatched. It was not universal, however, as it had to be made for each special lamp; but it had the redeeming feature that any one could readily make such a diagram on crosssection paper for any particular lamp and scope. This diagram was described by the writer in the Electrical Engineer of January, 1887, and is doubtless known to many, as it has since been copied and published by some one else in a well-known book on wiring, though without any acknowledgement of the true authorship. This diagram, however, is only a partial solution of the problem, as it performs only two of the three multiplications and 
divisions required by the formula ; it is necessary first to multiply arithmetically the number of lamps by the distance in feet, the diagram will then perform the remaining multiplication and division, both in one operation, giving as the result the size of the wire or the gauge number. To attempt to give on a regular scale the diameter of the wire as distinguished from the cross-section, would require a series of parabolas to be drawn; this would render it practically out of the question for any one to make the diagr m himself. The simple device was therefore resorted to, to use a regular arithmetic scale of cross-sections, instead of diameters or gauge numbers. All the lines then become straight diagonals and are therefore very easily drawn. Furthermore, the different factors which enter into the calculations were so placed on the diagram that the series of hyperbolas called for by the formula resolved themselves all into straight lines.

The objections to this method were that it required a different diagram for each different lamp, and that it did not multiply the number of lamps by the distance in feet. The object of the present diagram is to overcome these two objections. This was accomplished by the simple device of having three of these diagrams or sets of diagonals placed next to each other, as shown in the accompanying diagram, so that a line could be followed readily from one to the other, thus enabling a series of multiplications and divisions to be performed. The diagrams might have been superimposed on each other, but this would make a confusion of lines.

As this same principle of making a succession of calculations graphically may be applicable equally well to other formulæ besides that for wiring; it may not be out of place here to explain the principle before showing how this particular diagram is to be used. The general principle may be laid down that for any formula containing three variable quantities, one of which is the product or the quotient of the other two, the "curves" representing their relative values may always be represented by a series of straight diagonal lines drawn through the centre or zero point. Such a set of lines will therefore enable one to make any calculations graphically for that formula. For instance, horse power is equal to volts multiplied by amperes; the constant 746 does not concern us at present. A series of diagonal lines properly spaced will therefore give directly either the horse power, the volts or the amperes, when the other two are given. The curves 
may also be made hyperbolas, but it is evidently absurd to draw hyperbolas when straight lines answer just as well and are even more accurate, as they can be laid off and drawn with great precision. Nothwithstanding this, such diagrams are often published as hyperbolas. The difference lies in the dispasition of the scales. One scale is vertical, the other horizontal, and the diagonal lines (or the hyperbolas) each represent one unit (or number of units of the third scale. To make the "curves" straight lines, the diagonals must be made to represent one of the two quantities which is equal to the quotient of the other two, and not the one which is equal to the product of the other two, because the curves would then be hyperbolas. In the example given the diagonals must represent voits or amperes, but not horse powers. The constants in such formulæ affect only the positions of the diagonals; althongh they increase considerably the work of arithmetically calculating the results, they do not affect in the least the graphical calculations after the diagrams are once drawn. For this reason alone the graphical calculations are that much simpler.

Now, the general formula for wiring is as follows:

Cross-section $=\frac{\text { current for one lamp } \times \text { number of lamps } \times \text { distance } \times \text { constant }}{\text { loss in volts }}$

containing six quantities only, one of which is always constant, being equal to twice the mil foot resistance of copper, if the crosssection is in circular mils. As we have just seen, calculations involving three of these five quantities may readily be made graphically by means of a single set of diagonal lines. For instance, for any one make of lamp the current per lamp is a constant, and may therefore be combined with the other constant as one; if, furthermore, the number of lamps and distance be multiplied together mentally and then used as one quantity, lamp feet, there are then only three variable quantities to the formula, and calculations can then be made by one set of diagonals. This will give the diagram mentioned in the early part of this paper. But a universal diagram must be equally well applicable to any lamp; and as graphical calculations are so much simpler, more rapid, and less liable to errors than arithmetical ones, it is preferable to multiply the lamps by the feet graphically also. Both of these may be accomplished by splitting up this formula into smaller ones, each of which contains no more than three variable quantities. Each formula can then be calculated separately by a simple diagram, as described, thus permitting the whole formula to be calculated graphically. 
To do this, let the first diagram perform the calculation,

$$
x=\frac{\text { current for one lamp }}{\text { loss in volts. }}
$$

In which $x$ is a mere auxiliary quantity. Let a second similar diagram perform the next calculation :

$$
y=x \times \text { number of lamps ; }
$$

and a third diagram the final calculation:

\section{Cross-section $=y \times$ distance.}

The constant may be combined with any one of these, it is immaterial which one. This triple calculation may at first seem to complicate matters on account of the new quantities, $x$ and $y$. These, however, are easily eliminated by the simple device of placing the three diagrams together, side by side, in such a position that the two $x$ scales coincide, and similarly the two $y$ scales. By doing this, one has merely to pass directly from one set of diagonals to the next to perform the successive steps of the calculation, without being concerned about the intermediate auxiliary quantities. These intermediate quantities correspond, and are equal to the successive products or quotients which are obtained in the successive arithmetical multiplications and divisions of these five quantities in the formula, which cannot, of course, be eliminated in making the calculations arithmetically, showing another great advantage of the graphical methods. This particular method of performing the successive calculations is, of course, not limited to this formula, which is used here merely as an illustration. Any other formula which may be split up into smaller ones, each involving one multiplication or division, can be calculated graphically by the same method.

In the accompanying diagram the formula just given is represented graphically as described. It will be seen to consist of three right angles each with its set of diagonals and a common center. Each two right angles have one side in common which represent the quantities $x$ and $y$, and which thereby drop out of consideration. The lower left-hand diagonals represent loss in volts, the upper left hand, number of lamps, and the upper right hand, distances. The remaining two of the five variables are represented by the starting scale vertically below the center, which represents the amperage of a single lamp, and the last scule, to the right of the center, which represents the final result, namely, the cross section. The constant is included in any one of these three sets. 
To perform the graphical calculations, simply follow from one scile to the next on the cross-lined background up to the last scale, the cross-sections, which is the final result sought for. An example is worked ont and illustrated by the broken line on the diagram. Starting first with the amperage of the particular lamp, .775, on the first scale, follow to the left (see the broken line) to its intersection with the required volt diagonal, 1.0 volt; thence up to the next diagonals, the lamps, 10 lamps; thence to the right to the feet diagonals, 50 feet; thence down to the scale of cross-sections, where the reading is about 8200 circular mils, or about a No. 11 B. and S. wire; the whole operation requiring about as much time as it would take to start the arithmetical calculations.

The relative disposition of the scales is also of importance in such a diagram. For instance, the amperage of the lamp is generally the same for a great many computations; it has therefore been made the first scale, so that it can be eliminated from all those computations by drawing a horizontal line, say in red ink, through this value and then starting all the calculations from this line, thus saving that first part of the work. Next to the amperage of the lamp, the loss in volts is often the same for many calculations; the volt scale has therefore been made the second one, so that it also may be cut out of these calculations. To eliminate this part, draw a vertical red line through the intersection of the ampere and the volt lines. All such calculations are then started directly from the intersection of this line with the lamp scales. Finally, by the dotted lines drawn through the last scale, as shown representing the cross-sections of the standard wire gauge sizes, the last part of the work is also saved. The only operation left to be performed, therefore, is to follow a horizontal line from the number of lamps to the number of feet; it would hardly be possible to have it simpler. As the majority of calculations are made for the same amperage and loss, this represents the most frequent conditions under which the diagrams would be used.

The range of such a diagram can evidently be made very great by having several sets of numbers on the scales, each set being say ten times larger or smaller than the other, the position of the diagonals will remain the same. By an accidental coincidence the position of the dotted lines representing the B. and S. gauge numbers, will also remain very nearly the same for different scales 

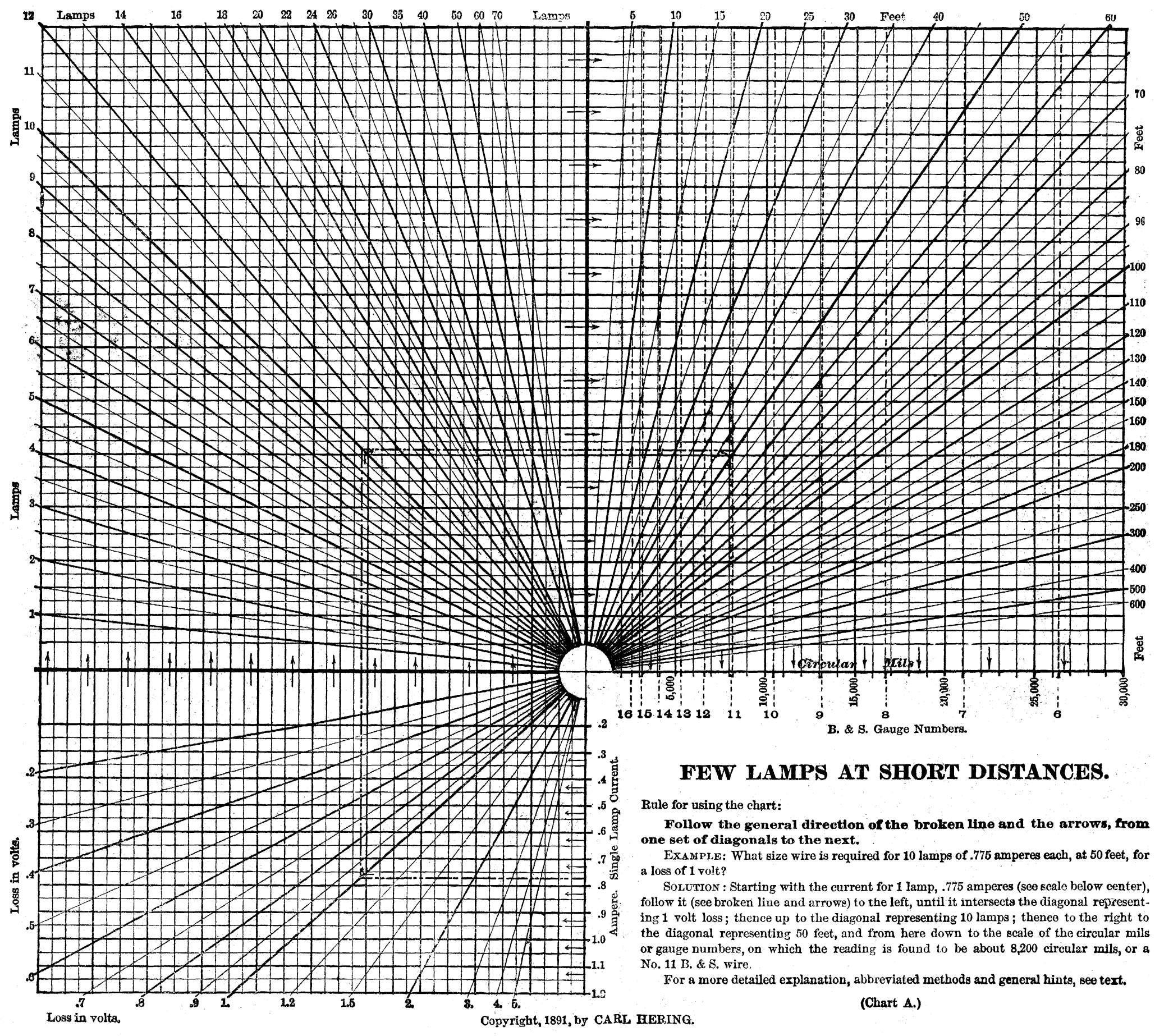
of circular mils, even though they represent different gauge numbers. Furthermore, lamps and feet are interchangeable on the diagram. Although the same diagram may have several different sets of numbers, it is probably preferable to have a separate dianram for each set. A set of three diagrams, of which the accompanying is the first one, will probably cover the usual ranges. For motor currents the same diagrams may be used, by assuming that the current represents that number of one-ampere lamps.

The whole diagram might have been made much smaller by superimposing the three parts over each other, instead of placing them next to each other, the principle remaining the same. The three centres of the diagonals would then be at the three corners of a rectangle. It was thought, however, that this might cause confusion, owing to the great multiplicity of crossing lines, and, therefore, be a disadvantage rather than an advantage.

There exists among many persons, unfortunately for themselves, a prejudice against any graphical methods of calculations. They appear to think that because such methods do not come within the narrow scope of their little multiplication tables, they must be something very difficult and complicated which would take too much time and trouble to master; and that such methods are suited only to professors and college men. In point of fact, graphical calculations are much simpler than ordinary multiplication, and very much simp'er than division. Graphical methods share this unwarranted prejudice with other short and simple methods of calculations, such as algebra, the slide rule, logarithms, etc. All such short methods carry their reward with them to such persons, and only to such, who take the small amount of trouble to master them. No one having many and complicated calculations to make, will ever regret it, if he spends a little time in becoming familiar with any or all of these short methods of calculating; it is his own loss if he does not take advantage of their existence.

\section{Discussion. $^{1}$}

Dr. F. A. C. Perrine:- Some five years ago I had occasion to make a diagram of a similar type to that of Mr. Hering's. While not quite so universal, I think it might be interesting to the association. (Making a sketch.) I number these horizontal lines which represent ten divisions in each, by the volts and the lines at right angles by amperes. I use 10 as being the most conveni-

1. By Messrs. Perrine, Hammer and Mailloux. 
ent. Then any diagonal drawn will be a line of constant resistance. If I also number the horizontal lines as distances, I use as convenient there 200 feet. Then, for calculating any size wire for a given distance-given loss, given amperage-I follow my line of amperes up until I come to the drop, and then follow the diagonal along to the line of proper distance in feet, and these vertical lines will correspond to the areas of the wire accordingly. Each division will correspond approximately to 20,100 circular mils and the whole method of using the table is simply to draw in a number of diagonals. If you are working at a constant, say five volts loss, then you draw in your five volt diagonals and you simply have your table. You take the amperage-say 20 amperes at 5 volts drop - that gives you that diagonal. For 800 feet you pass down the diagonal to this point, and drop down here, and this will give you the size of the wire in circular mils. Now, as all the wire manufacturers publish tables of circular milages as well as diameters, it gives you the size of wires practically, and taking 20,000 circular mils as being the distance of one of these spaces, it will give the small tenths equal to 2,000 circular mils, which is equal to about a No. 18 wire. It is, of course, exactly the same formula that Mr. Hering has used, with the exception that I have taken as my diagonals, lines of constant resistance rather than constant voltages of the lamps. As I say, I first started at this table about five years ago and it was only last week that I succeed $d$ in getting these numbers placed to my satisfaction. I had these two lines reversed for the past four years and it brings the area of all the wires that are ever used in about that space, but if you simply turn them round to bring the volts and distances on the same side, it enables you to use a 20 division cross-section paper up to a 410,000 circular mil wire, and if that is not large enough you can simply calculate your wire for half the distance and multiply by two. Then on the line leneath this I have also calculated the carrying capacities, so that once finding the wire of the proper size, you look under it and see if it has the proper carrying capacity. [Applause.]

The Chairman :- The Chair would like to state that Mr. Hering alluded to the fact that some time ago he published a table which has since been copied without giving credit to its author. I think that is a thing that has been very frequently done in electrical matters-in fact, much too frequently, and Mr. Hering's work in this line is one that has attracted universal attention, and certainly there has been no one who has given it more close attention. His reference to the diagram by Mr. Walstrom, which was made in 1884 - from a historical point of view-it calls to my mind a diagram that was made the year previous to that by $\mathrm{Mr}$. Fritsche of Berlin, the inventor of the Fritsche dynamo. It was not universal in its character, but in a couple of lines I can give you an idea of what it consisted of. I was during the years 1883 and 1584 connected with the Technical Department of the Ger- 
man Edison Company, with which Mr. Fritsche was connected as one of the engineers, and he took some tables which had been calculated for wiring leads and used only one type of incandescent lamp of one electro-motive force. His form of construction of this table, of which, by the way, I have one of the blue prints in my possession, which I will be very pleased to show to Mr. Hering, or others, was made in 1883. (Making sketch.) And the table was, of course, very similar in its character. You take an Edison incandescent lamp of 110 volts, and by taking this table and running the fingers up where these two lines meet, it would give us the circular mils of the wire for carrying a certain number of lamps a given distance. I think that was the first diagram of this nature that was made, and I take great pleasure in placing the credit for originating that in Mr. Fritsche's hands.

Mr. Marllodx:--I think that all of these methods really depend upon the same principle, which is the general principle of the curve stated in Mr. Hering's paper. The curve described by Dr. Perrine can be assimilated to the same thing. The only difference comes, as I understand, in the choice of the variable quantities which are ascribed to the different lines, and in his case he superposes two of the diagrams. If he had superposed a third, it seems to me that he would have arrived at the same result that Mr. Hering arrives at by putting the three systems of diagrams together. This method that you have just described, it seems to me, is only one of the same things, excepting that the circular mils in that case are represented by the diagonal lines, which is only another illustration of how the different lines may be used to represent at will different factors.

Mr. Hering :- I am very glad to hear of this diagram of Mr. Fritsche. An objection to it is that you have to follow two lines to their intersection. If you have a great many calculations to make, this is not as simple as following one line around a corner as in my diagram. The units in Mr. Fritsche's diagram could be changed about so that instead of following two lines you need follow only one.

The Chamman :--I might add that Mr. Fritsche had at periods placed dotted lines upon his chart, which assisted the eye in following, and for rough work they answer all purposes very nicely, and by following out the line the calculation could be arrived at instantly by placing the finger at the intersection of the dotted lines. If there is no further discussion on Mr. Hering's paper, we will proceed to a paper which is not down under the afternoon session. It is "A Thermo-Electric Method of Studying Cylinder Condensation in Stean Engine Cylinders," and I take pleasure in introducing to the society the author, Prof. Edwin H. Hall of Harvard University. 